\title{
An overview of Hydro Tasmania's dynamic real-time inflow prediction and flood forecasting system
}

\author{
$\underline{\text { K. Robinson }}{ }^{a}$, R. Parkyn ${ }^{b}$, F. Ling ${ }^{a}$ and S. Blundy \\ ${ }^{a}$ Entura, Hydro Tasmania, Tasmania \\ Email: Kim.Robinson@entura.com.au \\ ${ }^{b}$ Hydro Tasmania, Tasmania
}

\begin{abstract}
Hydro Tasmania manages 30 hydro-electric power stations fed from 45 major lakes across Tasmania, 13,500GL of water is managed annually. Forecasts play an important role in optimising the operation of the system, assisting in minimising spill, ensuring lakes operate at economic levels and that flood and environmental risks are managed. These forecasts are generated by Hydro Tasmania's Dynamic Real-time Inflow Prediction (DRIP) system which produces 7 day forecasts for 61 locations across Tasmania, 21 of which are then used by lake level prediction models.

In 2014 Hydro Tasmania initiated a project to upgrade this system with the aim of improving forecasts and gaining a better understanding of the forecast uncertainty. The project involves: implementation of gridded rainfall forecasts, a GIS based model rebuild and auto calibration. For the forecast points, the system now provides an improved best estimate forecast and, based on analysis of the rainfall forecast uncertainty, a high and low forecast.
\end{abstract}

This paper discusses how these forecasts are used to inform operational decisions, gives an overview of the model development process, operational implementation, and reflects on the strengths and weaknesses of the system.

Keywords: Decision support system, short term forecasting 
Robinson K, An overview of Hydro Tasmania's dynamic real-time inflow prediction and flood forecasting system

\section{BUSINESS DRIVERS FOR AN INFLOW FORECASTING SYSTEM}

Hydro Tasmania's DRIP inflow forecasting serves two aims: firstly to provide power station operators with forecast inflows; and secondly to provide inflows for flood warning purposes.

The operators' use of the forecasts is driven by economics. With foreknowledge of high inflows over the next seven days, operators are able to reduce spill, and hence increase over-all energy production, by tactically running power stations more in the period preceding higher inflows: this creates more "airspace" that can be used to store the subsequent high inflows. Alternatively, during moderate-inflow periods, the forecast can give more confidence that spill can be avoided. This confidence means that airspace can be reduced and hence that the "head effect" can be maximized, i.e. with higher lake levels, more energy is produced from the same water.

Another benefit during moderate-inflow periods is that the forecasts allow electricity production to be better timed into the market, and since market-price variations can be large, this can be of significant value.

During periods where little rain is forecast the operators focus more on meeting demand, while retaining capacity for unexpected high market-prices and maintaining sufficient storage for potential dry spells beyond the rainfall-forecast horizon. The models give an estimate of what inflows will support the storages, if any. However, it is fair to say that in these drier periods, when the inflows are on a recession curve, the models offer less additional value over-and-above simpler estimation methods. As the inflow-forecasts only go out seven days they are not capable of informing the requirement to retain water for extended dry periods: Hydro Tasmania uses a different modelling approach for that purpose.

The other key aim, to provide inflows for flood forecasting, allows Hydro Tasmania to fulfill its roles and responsibilities regarding managing high storage levels and high outflows. In essence, the DRIP inflows feed into storage-models that provide forward estimates of lake level and outflow. A set of alert-levels is included in the system, which raise alerts if the level or outflow forecasts exceed specified thresholds.

\section{OVERVIEW OF THE DRIP SYSTEM}

The Hydro Tasmania inflow forecasting system, DRIP, includes 22 rainfall-runoff models. Each of these is a node-and-link model of sub-catchments and river-channels (although the sub-area water-balance is based on AWBM). The models are run on a two-hourly cycle and use the currently available data from Hydro Tasmania's TimeStudio database. This includes: measured rainfalls and stream-flows (by Hydro Tasmania, these are available near-real-time); and forecast rainfall (by Bureau of Meteorology, BOM).

Because the models utilise live stream-flow and rainfall measurements, and these are routed downstream, the forecast flow at the model outlet is partly comprised of the effects of those measurements. However, experience has shown that their effects are very short-lived and that, for all but very short lead times, the forecast rainfall is the main determinant of forecast flow. This is partly because of the steep terrain being modelled and the short distances to hydro lakes. The net result is that, operationally, the largest source of uncertainty in the flow forecast is, by far, the forecast rainfall.

\section{MODEL DEVELOPMENT}

The following section discusses the approach taken to developing the models. Model development can be broken into three stages: model build, calibration time series derivation, and model calibration.

\subsection{Model Build}

To build such a large number of models a semi-automated approach was taken. A key benefit of this approach is that it ensures consistency between the models which has benefits for understanding performance and ease of maintenance. The procedure to develop the models was as follows:

1. The catchment model schema was defined in ArcGIS. This included sub catchment breakup, junction nodes, rain gauge information and the routing network.

2. The model schema was loaded into a model build interface. The build interface creates a generic model with all relevant rainfall runoff code, calibration code, rainfall inputs, model-health reporting and catchment rainfall calculations.

3. The generic model was customised as necessary, for example additions such as forecast output links, any specific operating or network rules, correction points at stream gauge sites and additional calibration points were made. Figure 1 gives an indication of the level of complexity of these rules. 
Robinson K, An overview of Hydro Tasmania's dynamic real-time inflow prediction and flood forecasting system

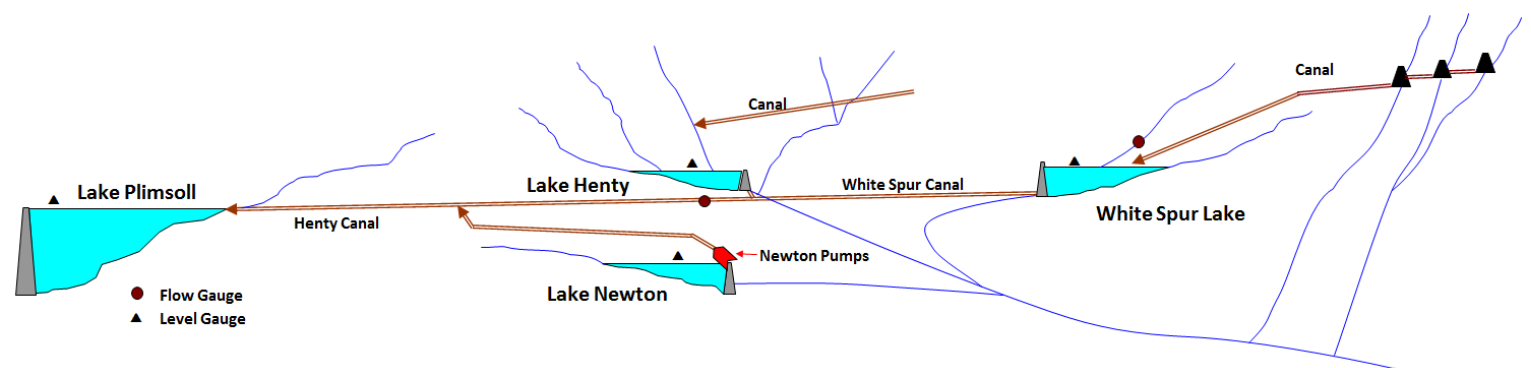

Figure 1. Schematic of the network that must be modelled, in addition to rainfall runoff modelling, to forecast inflows to Lake Plimsoll.

\subsection{Calibration time-series development}

Calibration time-series were derived to enable model calibration. Each catchment model was calibrated to either observed or derived flow at one or more points in the catchment. On a case by case basis practitioner judgment was used to assess which flow series was more appropriate. The decision on which flow series to use was based on factors such as: how well the catchment of the nearest stream gauge represented the forecast point catchment, and the quality of the derived flow record. The approach taken to deriving each of these record types is described below.

\section{Observed flow}

Hydro Tasmania has an ongoing quality control process in place for stream flow data which involves data managers reviewing and assigning quality codes to observations. This meant that quality codes could be used to exclude data that was not suitable for calibration, this included: estimated data, data that has been checked and found to be in error, poor data and data in error.

\section{Derived inflow}

Derived inflows are the natural pickup to a storage excluding discharge from storages upstream. Generally these were derived for the past 10 years (depending on data availability and quality) via the following process:

1. Timeseries of most non-natural inflows and outflows to Hydro Tasmania's storages are monitored and stored in TimeStudio. Applicable time series were identified by studying schematics of the scheme to identify all non-natural storage inflows and outflows.

2. A model was developed which calculated natural pickup by the following equation:

○ Natural Inflow = Delta Storage - Nonnatural inflows + Outflows.

The time series were calculated on an hourly time step. Sections where one or more of the inputs had a data gap of more than 2 hours were removed from the record,

3. A smoothing algorithm was applied (generally 6 hourly moving aggregate with -3 hour time offset) to smooth out jumps in time series,

4. The derived record was visually inspected and significant suspicious sections of the record were removed. This captured periods where incorrect instrument readings or non-monitored inflows/outflows to the storage affected the derived flow series.

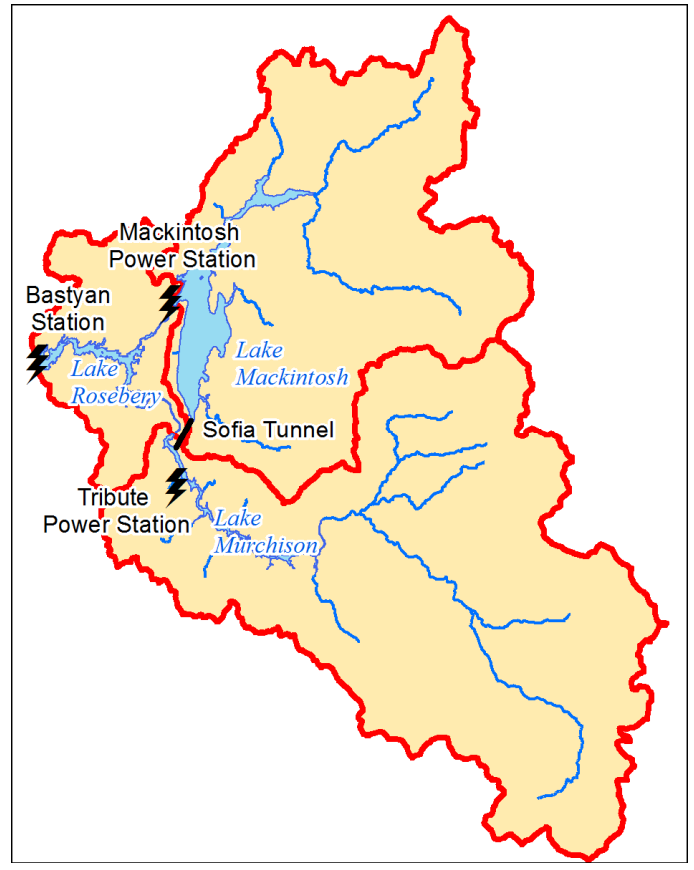

Figure 2. Highlights the elements that need to be accounted for when deriving natural inflow for some of the storages in the Pieman catchment.

This process is sensitive to instrumentation accuracy and rating curves. It is more effective for storages with pickup flows that are large compared to pass-through flows and where the lake surface area was small compared to the catchment. For example, for the areas 
Robinson K, An overview of Hydro Tasmania's dynamic real-time inflow prediction and flood forecasting system

shown in Figure 2, Lake Murchison's inflow derivation was excellent and Mackintosh's was useable. Conversely no useful inflow signal could be derived for Lake Roseberry because the smaller natural-inflow signal is lost amongst the high pass-through flows from Murchison and Mackintosh (Figure 3).

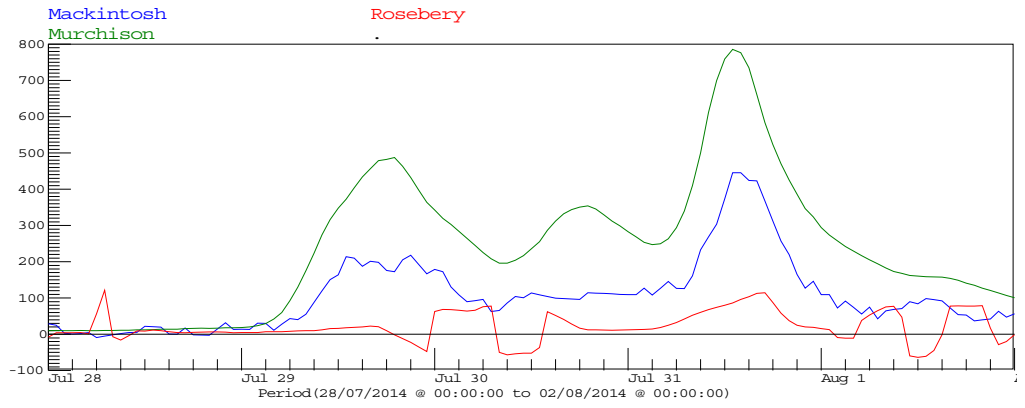

Figure 3. Example hydrographs of inflows derived for storages in the Pieman catchment.

\subsection{Model calibration}

The model parameters which transform rainfall to inflows were calibrated using the calibration time series.

The objective function used in calibration was Nash-Sutcliffe Efficiency which is a common method used to assess hydrologic model performance (WMO 2011). The coefficient was maximised over the calibration period, which generally extended for 10 years.

Cross validation of the calibration performance over an independent period was not carried out. It was considered that the additional effort in doing this would not add significant benefit to understanding the model performance, particularly given that a relatively long period of record was used in calibration and the other uncertainties at play in the model forecast performance.

The parameters of each model were calibrated using a global optimization algorithm called Shuffled Complex Evolution (SCE). An implementation of SCE developed by(Cohen 2012) in R for WISKI Modelling was utilised. The SCE algorithm applies various model parameter combinations to meet an objective function, in this case NSE. The models utilise Australian Water Balance Model 2 Tap (Parkyn \& Wilson 1997), catchment and channel routing, and parameters for each of these functions were optimised in calibration. The configuration of the SCE algorithm was guided by (Duan et al. 1993).

\section{OPERATIONAL IMPLEMENTATION AND FORECAST PERFORMANCE}

In operation the models utilise a network of telemetered rainfall gauges, located within or near the catchment, to forecast inflows. For all future time steps, those measured rainfalls are substituted with rainfall forecasts from the Bureau of Meteorology.

The entire process is automated; from the collection of input data, running of forecast models, through to display of results. This automated process is triggered by Hydstra TSM Scheduler, which activates routine tasks. Each model is operated at 2-hourly intervals and forecasts 7 days into the future. A data management duty officer is on call 24 hours a day to support and maintain the scheduler machines.

Naturally, there is a reduction in forecast accuracy with increasing lead-time. The most accurate forecasts are based on measured flow data, but this gives very little warning time as the flows are already in the river. The models also transmit measured rainfall inputs, which gives longer warning times but also additional uncertainty. The longest warning times are achieved using forecast rainfall data as input to hydrologic models, but these forecasts are the most uncertain.

The key sources of forecast uncertainty stem from (in decreasing order of importance): rainfall forecast error, calibrated model performance, uncertainties in observed data, methods of correction to observed streamflow, and to a lesser degree uncertainties in rating curves which transform observed parameters to flow. A description of the impact of each of these is provided in the following section. 
Robinson K, An overview of Hydro Tasmania's dynamic real-time inflow prediction and flood forecasting system

\subsection{Rainfall forecast performance}

The Bureau of Meteorology's ADFD forecasts are used as rainfall forecast input to the models. The forecasts cover the entire Australian domain and are available in a gridded format, with approximately $3 \mathrm{~km}$ resolution across Tasmania. The ADFD files are updated routinely at approximately $6 \mathrm{am}$ and $6 \mathrm{pm}$ local time and can also be updated at other intervals. Further information about the ADFD is available in (Bureau of Meteorology 2015).

The two data grids used in this system are the $25 \%$ probability of exceedance (POE) daily rainfall and the 3hourly average rainfall amount. The daily forecast extends to 7 days into the future and the 3 hourly forecast extends 2 days into the future. A 50\% POE daily rainfall product is also available however an assessment of its performance demonstrated that it is less representative of observed rainfall than the $25 \% \mathrm{POE}$.

Data comparisons between the ADFD forecasts and observed rainfall found that in general the forecasts contained biases. Therefore a process was established to bias correct the forecasts and calculate the uncertainty. This bias correction allows for local issues that aren't adequately modelled by the forecast process, such as elevation and aspect, which can significantly alter observed rainfall at the gauge, and secondly, any inherent bias in the BoM forecasts.

Forecast performance and bias correction functions were derived by analysing forecasts issued between March 2013 and October 2014. The observed vs forecast data was normalised on Box Cox space, a linear regression modelled fitted and the $50 \%$ prediction interval calculated. An example for Gwendy Lake site is shown in Figure 4.

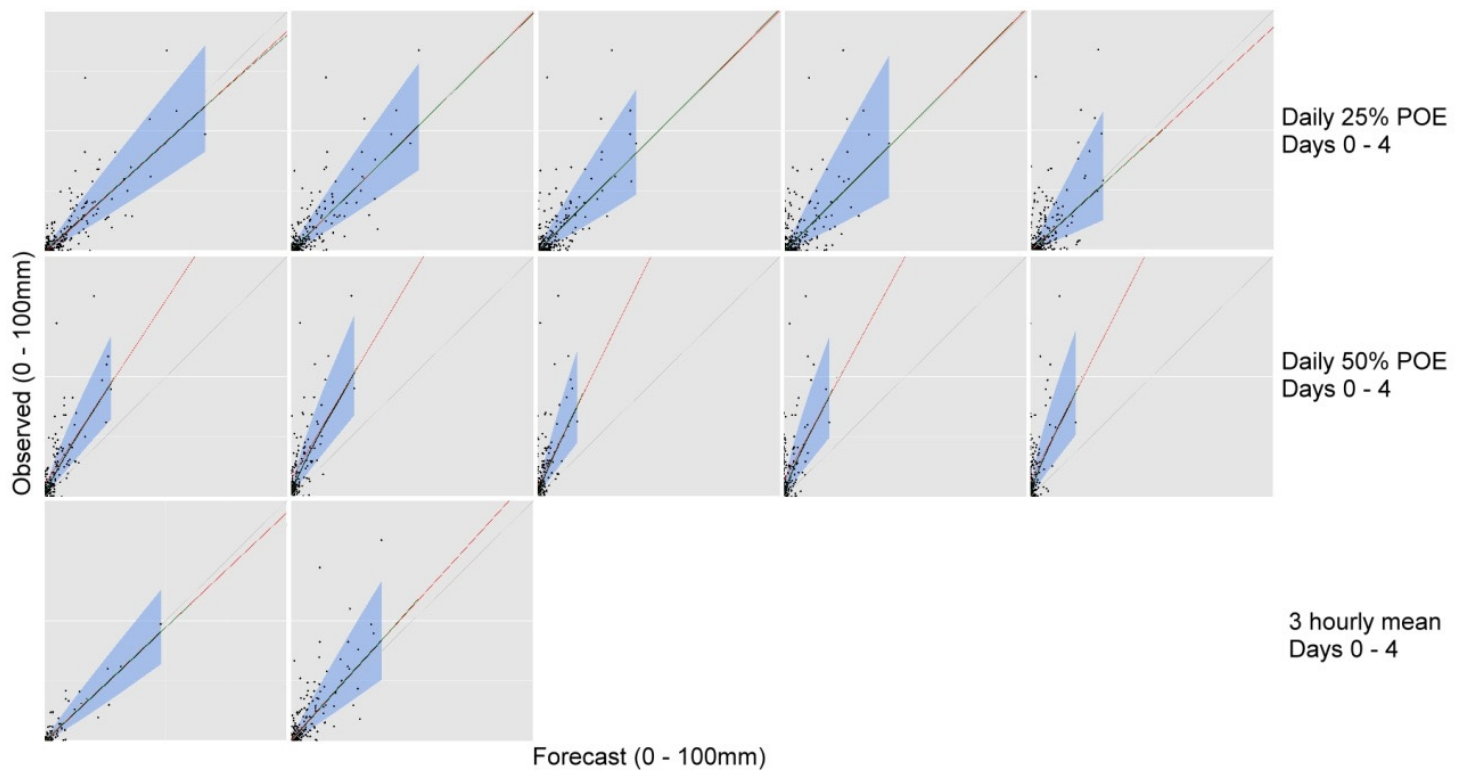

Figure 4. Scatter plots of forecast performance at Gwendy Lake. These demonstrate the tendency for the 50\% POE to under predict rainfall depths across all lead times and the increasing uncertainty of all forecasts with with lead time.

In addition to the primary inflow forecast, high and low model models are also run. These aim to communicate the impact rainfall forecast uncertainty has on the model results. They are based on the upper and lower bounds of the day-1 rainfall forecast $50 \%$ prediction interval.

\subsection{Calibrated model performance}

Model calibration performance was assessed at all calibration and forecast points, by running the models with observed rainfall inputs. Nash-Sutcliffe Efficiency (NSE) was calculated across the whole period of record and the full range of flows. Models with a higher NSE will generally perform better than models with a lower NSE however, NSE does not capture volume biases, or the model's ability to capture events within particular flow ranges or seasons.

For the majority of the models NSE was calculated at the time step of interest, which is hourly, and ranged from 0.76 to 0.92 which is considered very good (Moriasi et al. 2007). For some forecast points the model 
Robinson K, An overview of Hydro Tasmania's dynamic real-time inflow prediction and flood forecasting system

performed poorly at an hourly time step - this typically occurred where there was a high uncertainty in the calibration time series. In these cases the reporting time step was increased until a satisfactory NSE was achieved. The aim of increasing the analysis interval was to check that, for longer intervals, the model would give a useful representation of the observed record, and hence indicated some skill at volume prediction over such intervals. The plot below shows the model performance for Lake Murchison inflow which achieved an NSE of 0.92 ; this is one of the better performing models.
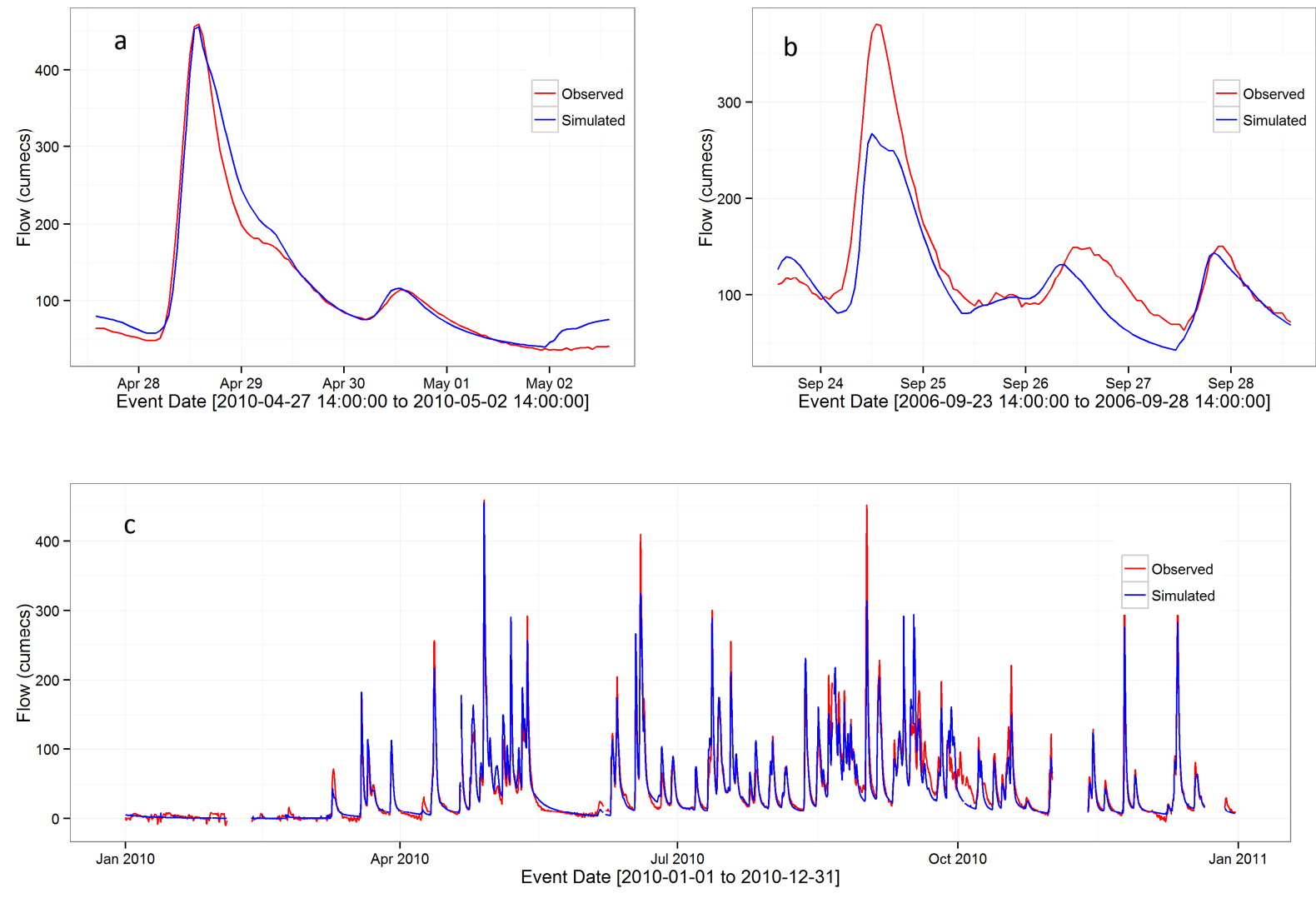

Figure 5. Hydrographs with modelled inflows (blue) and observed flows (red) for Lake Murchison inflow for two annual maximum events with a) good model performance, b) poor model performance, and c) an entire year's record at an hourly time step (2010).

\subsection{Observational uncertainties}

The forecasting process is automated from data collection through to forecast generation. Handling of observational uncertainties is therefore an important aspect of the DRIP system. For example, spikes or null periods in observed data will have a big impact on forecast results if not handled correctly. To minimise the likelihood and impact of erroneous observed data a comprehensive hydrographic maintenance program is in place along with complementary quality assurance and control processes. These processes automatically carryout tasks such as; clearing out unrealistic spikes in the observed data, infill missing values with supplementary sites, and notify personnel if data is not arriving in a timely manner.

\subsection{Other uncertainties}

Many of the models are calibrated to derived flow series whose accuracy is dependent on rating curves such as power station discharge, spillway discharge and lake level to volume curves. While these ratings are derived using best practice techniques they are generally not as accurate as established stream gauging techniques. These errors in some cases can result in the modelled inflows having systematic biases when compared to actual inflows. This situation is actually preferable as the modelled results are fed through downstream systems which use the same rating curves to plan system operation, this means that the errors balance out. 
Robinson K, An overview of Hydro Tasmania's dynamic real-time inflow prediction and flood forecasting system

In summary, forecast uncertainty is variable depending on all of the above. If there is no rain then model uncertainty is the biggest factor but generally the models are most heavily relied upon for 1-2 day planning when rain is on the horizon - in this space their results are most dependent on the rainfall forecast.

\section{STRENGTHS AND WEAKNESSES OF THE SYSTEM}

This paper has provided an overview of Hydro Tasmania's inflow forecasting system. A wide range of techniques have been adopted to develop and implement the system, some of them more successful than others.

Adopting a semi-automated approach to model development and calibration has ensured the models have a consistent coding structure. This approach increased development productivity and the system is now easier to support than if the models were developed individually. Having the same calibration metric means that all models have similar strengths and weaknesses at modelling the inflow hydrograph, this makes it easier for users to understand how well the models perform under different conditions.

The approach to developing calibration time-series was most successful for storages with relatively small surface area to catchment ratios, and with a large local pickup compared to pass through flows.

The entirely automated nature of the system has been very successful. Modern day observation and telemetry techniques have proved very reliable. This combined with auto QAQC algorithms and model scheduling systems means that failure-to-issue, and critically erroneous forecasts, are virtually non-existent.

Handling and communication of model uncertainty is the system's biggest weakness. The biggest source of uncertainty comes from the rainfall forecasts and the current approach to handling this does not account for the increasing uncertainty with lead time or variable uncertainty associated with forecasting a particular weather system type.

\section{REFERENCES}

Bureau of Meteorology, 2015. Australian Digital Forecast Database (ADFD) User Guide, Bureau of Meteorology. Available at: http://www.bom.gov.au/catalogue/adfdUserGuide.pdf.

Cohen, W., 2012. Hydrologic Model Parameter Optimisation. University of Tasmania.

Duan, Q.Y., Gupta, V.K. \& Sorooshian, S., 1993. Shuffled complex evolution approach for effective and efficient global minimization. Journal of Optimization Theory and Applications, 76(3), pp.501-521.

Moriasi, D.N., Arnold, J.G. \& Van Liew, M.W., 2007. Model evaluation guidelines for systematic quantification of accuracy in watershed simulations. Transactions of the American Society of Agricultural and Biological Engineers, 50(3), pp.885-900. Available at: http://swat.tamu.edu/media/1312/moriasimodeleval.pdf.

Parkyn, R. \& Wilson, D., 1997. Real-Time Modelling of the Tributary Inflows to ECNZ's Waikato Storages. Hydrology and Water Resources Symposium, pp.445-449.

WMO, 2011. WMO-No. 1072 Manual on flood forecasting and warning, World Meteorological Organization. 